\title{
IVD.23 - Comparative study of in situ hybridization and quantitative PCR for diagnosis of cutaneous infection by Leishmania infantum in dogs
}

\author{
Ricardo Gonçalves Silva ${ }^{1 *}$; Sandro Antônio Pereira ${ }^{2}$; Natália Pedra Gonçalves; Greice Maria \\ Silva da Conceição ${ }^{1}$; Luíz Cláudio Ferreira²; Fabiano Borges Figueiredo³ \\ Menezes ${ }^{2}$. \\ 1Fiocruz/Bio-Manguinhos; \\ 2Fiocruz/INI; \\ 3Fiocruz - Paraná.
}

Introduction: The zoonotic visceral leishmaniasis (ZVL) is a disease caused by the protozoan Leishmania infantum and of great importance in public health in Brazil, which affects humans, domestic and wild mammals. The diagnosis of L. infantum infection in dogs is performed by laboratory techniques, which must be accurate, because these animals are the main source of infection for the biological vector in the urban environment. Formalin-fixed paraffinembedded skin samples (FFPE) are frequently used in laboratorial routine for the diagnosis of infection by L. infantum in dogs, because they are a good target and do not need ideal conditions of cooling and transportation in the field. These are often the only samples available for the diagnosis of Leishmania spp. In these cases, the diagnosis at species level is only possible by in situ hybridization (ISH) and PCR techniques, as quantitative PCR (qPCR). However, the sensitivity of these techniques is little known.

Objective: To determine the sensitivity of ISH and qPCR in FFPE skin samples for the diagnosis of L. infantum infection in dogs using skin samples positive by parasitological culture (PC) as the reference standard.

Methodology: FFPE skin samples of 48 dogs (27 with clinical signs and 21 without clinical signs) having cutaneous infection by L. infantum confirmed by PC and by multilocus enzyme electrophoresis were examined by ISH and qPCR using specific probes for L. infantum. These samples were collected by biopsy of healthy skin of scapula in dogs from different endemic areas of ZVL in Brazil, between 2008 and 2015. The ISH technique was examined by two observers with different experiences in light microscopy.

Results: The sensitivities of qPCR and ISH were, respectively, $77.0 \%$ and $58.0 \%$. The sensitivities of qPCR in dogs with and without clinical signs were, respectively, $70.4 \%$ and $85.7 \%$. The sensitivities of ISH in dogs with and without clinical signs were, respectively, 55.5\% and 62.0\%. The interobservers agreement for the ISH was fair (Kappa=0.32). The ISH presented sensitivity directly associated with parasitic load quantified of L. infantum in the skin

Conclusion: The ISH and qPCR showed satisfactory sensitivities for the diagnosis of $L$. infantum in FFPE skin samples of dogs, even in dogs without clinical signs, being qPCR the most sensitive technique. When used together, these two techniques increase the sensitivity for L. infantum diagnosis in the FFPE skin samples of dogs and allow the identification of active infections by this parasite.

Keywords: Canine Visceral leishmaniasis; in situ hybridization; quantitative PCR 